\title{
A NEW OUTFLOW BOUNDARY CONDITION
}

\author{
T. C. PAPANASTASIOU, N. MALAMATARIS AND K. ELLWOOD \\ Department of Chemical Engineering, The University of Michigan, Ann Arbor, MI 48109. USA.
}

\begin{abstract}
SUMMARY
Boundary conditions come from Nature. Therefore these conditions exist at natural boundaries. Often, owing to limitations in computing power and means, large domains are truncated and confined between artificial synthetic boundaries. Then the required boundary conditions there cannot be provided naturally and there is a need to fabricate them by intuition, experience, asymptotic behaviour and numerical experimentation. In this work several kinds of outflow boundary conditions, including essential, natural and free boundar conditions, are evaluated for two flow and heat transfer model problems. A new outflow boundary condition, called hereafter the free boundary condition, is introduced and tested. This free boundary condition is equivalent to extending the validity of the weak form of the governing equations to the synthetic outflow instead of replacing them there with unknown essential or natural boundary conditions. In the limit of zero Reynolds number the free boundary condition minimizes the energy functional among all possible choices of outflow boundary conditions. A review of results from applications of the same boundary conditions to several other flow situations is also presented and discussed.
\end{abstract}

KEY WORDS Open boundary conditions Backward-facing step Unbounded fow Free boundary condition

\section{INTRODUCTION}

In order to solve differential equations on finite domains, boundary conditions are needed along the entire boundary or parts of the boundary, depending on the nature of the differential equations-hyperbolic, parabolic, elliptic. ${ }^{1}$ These boundary conditions are provided by the physics when the finite domain is the actual physical domain, e.g. flow in a cavity, heat transfer and reaction in a closed reactor, loading of finite solids. Infinitely large physical domains often need to truncated to synthetic, artificial finite domains because of limitations in computational speed and time, e.g. flow in long converging channels, slowly decaying thin films, heat and mass transfer through infinite and semi-infinite solids. For these problems there is an uncertainty about the appropriate boundary conditions at the synthetic boundaries because the physics is not known there. This poses significant computational difficulties for even relatively well-behaving steady systems. These difficulties grow beyond control for unsteady and/or unstable systems.

A case of instance is shown in Figure 1(a), where a thin film flowing down a long inclined plate goes unstable for one reason or another. Boundary conditions at all times are well defined along the real boundary, which consists of a chosen inlet, the free surface and the solid wall. In contrast, no boundary conditions can be specified at the synthetic outlet for an unsymmetric travelling wave, which is the most general case: for example, either a zero free surface slope or a specified free surface elevation at the synthetic outflow may impose artificial constraints not necessarily corresponding to the local physics; nor can any information from the downstream behaviour be utilized to derive asymptotic boundary conditions because the downstream behaviour is unknown and hardly predictable. To address problems involving synthetic boundaries, appropriate 


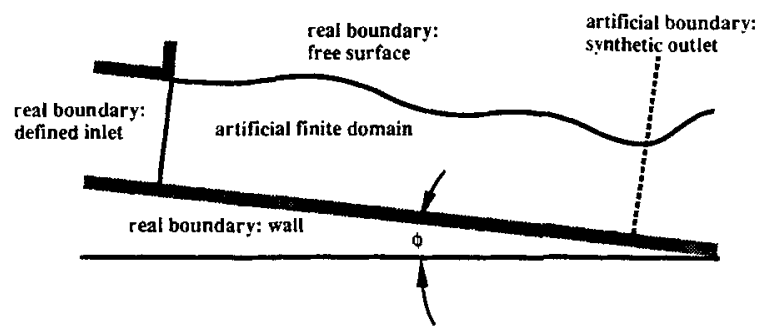

(a)

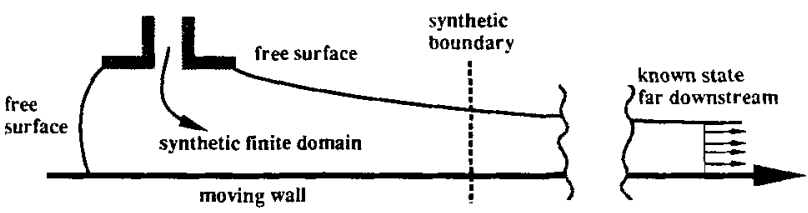

(b)

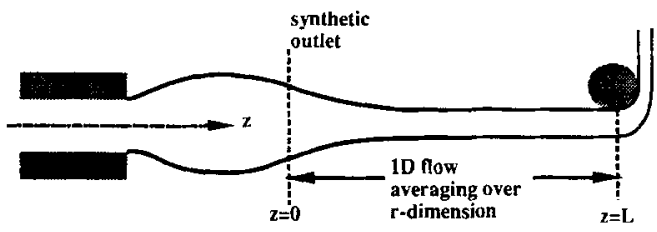

(c)

Figure 1. Several kinds of unbounded flows: (a) unstable thin film; (b) exponentially decaying thin film; (c) axisymmetric fibre spinning

boundary conditions that can mimic or even not alter the local behaviour need to be found by numerical experimentation and comparison of predictions with experimental evidence or with asymptotic and/or analytic solutions.

Open boundary conditions are applied at synthetic outflows (and for that matter at synthetic inflows) of truncated domains in order to allow phenomena generated in the domain of interest to pass through the synthetic boundary without undergoing significant distortion and without influencing the interior solution. These conditions received attention initially with hyperbolic problems common in oceanography (ocean circulation, eddies, etc.) and meterology (convection, weather prediction, air pollution modelling, etc.). They were accommodated by Sommerfeld radiation conditions, by one-side differencing methods and by viscous damping at these boundaries. Among them, the Sommerfeld condition formulated as

$$
\frac{\partial \phi}{\partial t}+c \frac{\partial \phi}{\partial x}=0
$$

for a quantity $\phi$ at the synthetic outflow propagating at phase velocity $c$ was followed, evaluated and developed further by several investigators. ${ }^{2-5}$ These conditions remain primarily associated with hyperbolic equations solved by finite differences and, unless an asymptotic behaviour downstream is known, ${ }^{3}$ they appear to work only in certain cases and for certain types of 
propagating waves. $^{4}$ Nevertheless, the Sommerfeld boundary condition, along the lines developed in Reference 2, remains the most widely used open boundary condition.

The simplest cases of flows with synthetic domains involve synthetic boundaries downstream whose behaviour can be reasonably approximated by perturbation techniques and asymptotic analysis of the equations between the synthetic boundary and far downstream where things are settled. A characteristics case is the exponential decay of thin films to planar films far downstream, as analysed by Higgins. ${ }^{6}$ The so-called non-reflective boundary conditions are constructed along the same lines. Figure 1(b) illustrates a case amenable to this kind of approximation.

A detailed evaluation of open boundary conditions in developing channel flows and thin film coating flows was conducted by Bixler. ${ }^{7}$ The most accurate condition was the mixed condition-also called the Robin condition-which related the behaviour far downstream to that at the synthetic outflow by asymptotic analysis. The traction and free surface inclination boundary conditions - also called Neumann conditions-gave satisfactory results too. The least accurate boundary condition - also called the Dirichlet condition-was the specification of the variables at the synthetic outflow. All the above boundary conditions-Robin, Neumann and Dirichlet - are made possible by the fact that the flow downstream from the synthetic outflow is in principle amenable to asymptotic analysis. However, in cases where asymptotic analysis is impossible, none of these conditions should be applied, because neither the exact values of the variables nor their derivatives are known and neither can their relation to the behaviour far downstream be found in any asymptotic way. A slightly different approach is to match the threeand the two-dimensional domains to two- and one-dimensional domains respectively downstream from a synthetic boundary, as suggested by Kistler and Scriven ${ }^{8}$ and shown in Figure 1(c).

The approach taken for flows similar to those of Figures 1(b) and 1(c) requires some knowledge of the behaviour downstream from the synthetic boundary and therefore does not apply to flows similar to those of Figure 1(a). For the latter class of flows an alternative approach was found to work reasonably well for several flow situations examined, as follows. Consider the flow of a Maxwell viscoelastic liquid in the one-dimensional (averaged) domain of Figure 1(c) alone. The governing equations are $^{9}$

$$
\begin{gathered}
\frac{\mathrm{d}}{\mathrm{d} z}\left(\frac{\tau_{z z}-\tau_{r r}}{u_{z}}\right)=0 \\
\tau_{z z}-\lambda\left(2 \tau_{z z} \frac{\mathrm{d} u_{z}}{\mathrm{~d} z}-u_{z} \frac{\mathrm{d} \tau_{z z}}{\mathrm{~d} z}\right)=2 \frac{\mathrm{d} u_{z}}{\mathrm{~d} z}, \\
\tau_{r r}+\lambda\left(\tau_{r r} \frac{\mathrm{d} u_{z}}{\mathrm{~d} z}+u_{z} \frac{\mathrm{d} \tau_{r r}}{\mathrm{~d} z}\right)=-\frac{\mathrm{d} u_{z}}{\mathrm{~d} z},
\end{gathered}
$$

where $\lambda$ is the relaxation time of the viscoelastic liquid and $u_{z}, \tau_{z z}$ and $\tau_{r r}$ are the $z$-dependent axial velocity, axial stress and radial stress respectively on the one-dimensional domain $0 \leqslant z \leqslant L$. To solve this one-dimensional problem, one would need boundary conditions on both the velocity and the stress at the synthetic inlet $z=0$. The difficulty here comes from the fact that the exact values of the stresses are unknown at the synthetic inlet; thus the imposition of arbitrary values of stress results in an artificial stress boundary layer downstream from $z=0$, as demonstrated in Reference 9. This difficulty was alleviated by not imposing any stress boundary condition at the synthetic inlet at all or, equivalently, by extending the validity of the weak forms of equations (3) and (4) to the inlet instead of replacing them there by arbitrary stress boundary values. Not only did the Newton iteration of the finite element equations converge quadratically, but the converged 
solution gave the appropriate values of the stress at the synthetic inlet as well; these stresses were validated a posteriori by re-solving the same problem with these stresses specified as boundary conditions, which eliminated the otherwise induced artificial stress boundary layer. It was concluded that the use of the weak forms of equations (3) and (4) as boundary conditions resulted in a well-posed non-singular problem, as evidenced by the converged solution and its accuracy.

The same idea was tried successfully in several other flows on synthetic (truncated) domains, including lubricated squeezing flow of Newtonian liquids, ${ }^{10}$ rotating thin films of Newtonian and shear-thinning liquids ${ }^{11}$ and channel flow of viscoelastic liquids. ${ }^{12}$ In this work this idea is further evaluated for two prototype flows and geometries which were topics of the Minisymposium on Open Boundary Conditions held at Stanford, CA on 14 July 1991 prior to the Seventh International Conference on Numerical Methods in Laminar and Turbulent Flow.

\section{GOVERNING EQUATIONS}

The flow geometries are shown in Figure 2. In Figure 2(b) an incompressible Newtonian fluid enters the upper half of a rectilinear channel (stratified backward-facing step, SBFS). As the flow progresses, the fluid is rearranged to a parallel flow at the outflow of the long domain after some eddy formation in the interior of the channel. The temperature of the fluid is kept constant along the floor and along the ceiling of the channel, where also no slip and no mass penetration are assumed. The governing equations of the SBFS problem at steady state are

$$
\begin{aligned}
\nabla \cdot \mathbf{u} & =0, \\
\mathbf{u} \cdot \nabla \mathbf{u} & =-\nabla p+\frac{1}{R e} \nabla^{2} \mathbf{u}-\frac{1}{F r} \mathbf{k} T, \\
\mathbf{u} \cdot \nabla T & =\frac{1}{P e} \nabla^{2} T .
\end{aligned}
$$

Here $\mathbf{u}=(u, v)$ is the velocity vector of the fluid, with $u$ and $v$ its components in the $x$ - and $y$-directions respectively, $p$ is the pressure, $T$ is the temperature and $\mathbf{k}$ is the unit vector in the
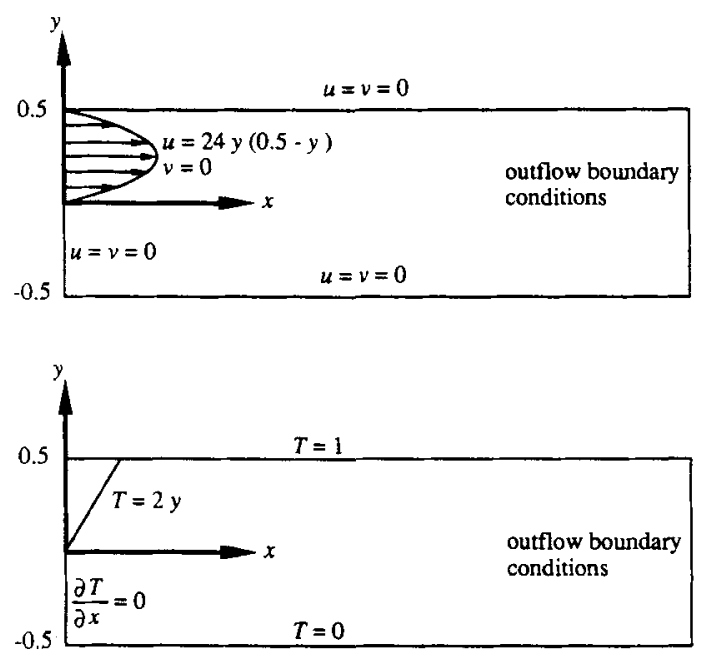

Figure 2. Domain and boundary conditions for (a) backward-facing step (BFS) and (b) stratified backward-facing step (SBFS) problems 
direction of gravity (opposite to the $y$-direction). $R e=u_{0} L / v$ is the Reynolds number, with $u_{0}$ and $L$ a characteristics velocity and length respectively and $v$ the kinematic viscosity of the fluid. $P e=u_{0} L / a$ is the Peclet number, with $a$ the thermal diffusivity of the fluid. $F r \equiv\left(u_{0} / u_{\mathrm{B}}\right)^{2}$ is the Froude number, with $u_{\mathrm{B}}$, the buoyancy velocity, defined as $u_{\mathrm{B}}=\sqrt{ }(\gamma g \Delta T L)$, where $\Delta T$ is the maximum temperature difference, $\gamma$ is the volumetric expansion coefficient and $g$ is the acceleration due to gravity. The Peclet number is related to the Reynolds number $P e \equiv P r R e$, where $P r=v / \kappa$ is the Prandtl number, with $\kappa$ the thermal diffusivity. In Figure 2(a) the isothermal case of the previous flow is analysed (isothermal backward-facing step, BFS). The governing equations of the BFS problem are equations (5) and (6), with the Froude number set to infinity in equation (6). Hereafter, short domain refers to a domain truncated to seven units of width from the inlet and long domain refers to a truncated domain of at least 15 width units.

\section{BOUNDARY CONDITIONS}

The boundary conditions at the physical (real) boundaries (Figure 2) are

$$
\begin{aligned}
x=0,-0.5 \leq y<0: \\
u=0, \\
v=0, \\
\partial T / \partial x=0 ;
\end{aligned}
$$

$x=0,0 \leq y \leq 0 \cdot 5$

$$
\begin{aligned}
& u=24 y(0 \cdot 5-y), \\
& v=0 \\
& T=2 y
\end{aligned}
$$

$x, y=-0 \cdot 5$ :

$$
\begin{aligned}
& u=0, \\
& v=0, \\
& T=0
\end{aligned}
$$

$x, y=0 \cdot 5$ :

$$
\begin{aligned}
& u=0, \\
& v=0, \\
& T=1
\end{aligned}
$$

The boundary conditions (10), (13), (16) and (19) were applied for the SBFS problem alone.

At the synthetic outflow boundary of the domain the following boundary conditions were examined:

(a) fully developed flow of

$$
u=-3 y^{2}+0 \cdot 75
$$




$$
\begin{aligned}
v & =0 \\
\mathrm{~d} T / \mathrm{d} x & =0 \quad \text { (only for the SBFS problem); }
\end{aligned}
$$

(b) free boundary condition, by simply evaluating the surface integrals of the momentum and energy equations in terms of the, as of yet, unknown outflow nodal values of $u, v, p$ and $T$ along with the volume integrals. This is equivalent to extending the weighted momentum and energy equations to the synthetic exit, as explained below. The specification of a datum pressure $p=0$ by means of the continuity equation at a unique node of the synthetic outflow was often found to improve convergence.

\section{FINITE ELEMENT FORMULATION}

The long flow domain was tesselated into the $(58 \times 18)$ rectangular finite elements shown in Figure 3, which produced solutions unchanged with further refinement. The unknown velocities $u$ and $v$, the pressure $p$ and the temperatue $T$ were expanded in terms of Galerkin basis functions as

$$
u=\sum_{i=1}^{9} u_{i} \phi^{i}, \quad v=\sum_{i=1}^{9} v_{i} \phi^{i}, \quad p=\sum_{i=1}^{4} p_{i} \psi^{i}, \quad T=\sum_{i=1}^{9} T_{i} \phi^{i},
$$

where $\phi^{i}$ are biquadratic and $\psi^{i}$ bilinear basis functions. The governing equations, weighted integrally with the basis functions, resulted in the following continuity, $R_{\mathrm{C}}^{i}$, momentum, $R_{\mathrm{M}}^{i}$, and enegy, $R_{\mathrm{E}}^{i}$, residuals:

$$
\begin{aligned}
& R_{\mathrm{C}}^{i}=\int_{V} \nabla \cdot \mathbf{u} \psi^{i} \mathrm{~d} V=0, \\
& R_{\mathrm{M}}^{i}=\int_{V}\left[\mathbf{u} \cdot \nabla \mathbf{u}+\frac{1}{F r} \mathbf{k} T-\nabla \cdot\left(-p \mathbf{I}+\frac{1}{R e} \mathbf{T}\right)\right] \phi^{i} \mathrm{~d} V=0,
\end{aligned}
$$

(a)

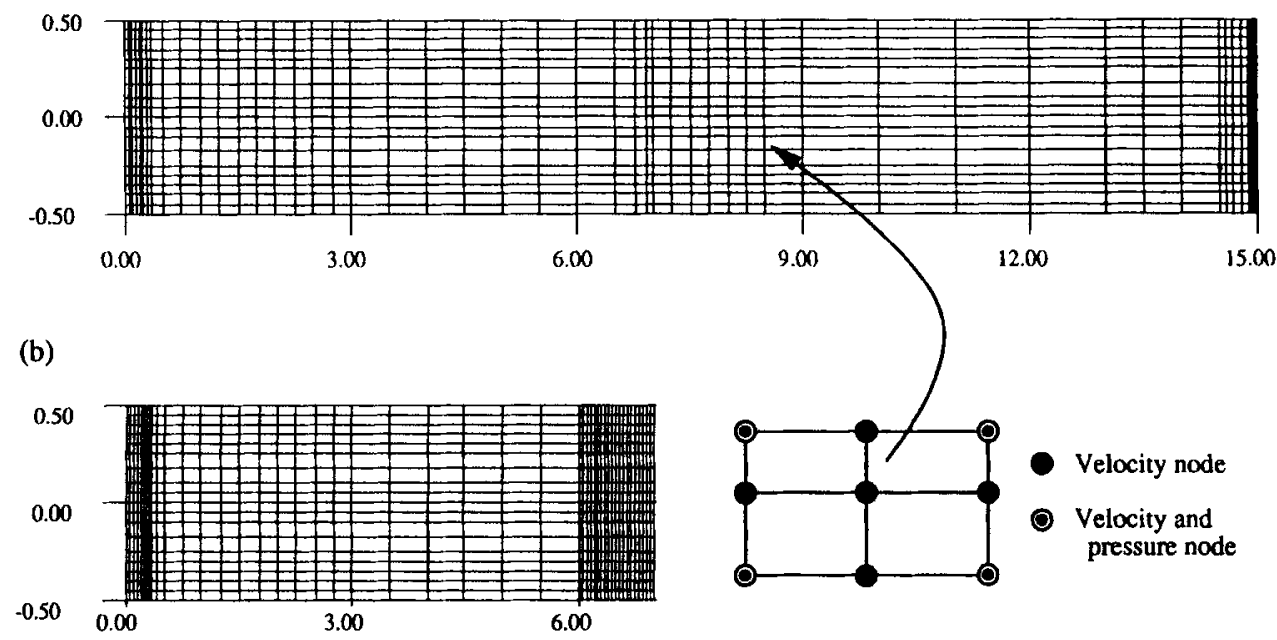

Figure 3. (a) Typical finite element tesselation used in this study and (b) refined short computational domain 


$$
R_{\mathrm{E}}^{i}=\int_{V}\left(\mathrm{u} \cdot \nabla T-\frac{1}{P e} \nabla \cdot \nabla T\right) \phi^{i} \mathrm{~d} V=0 .
$$

Here $\mathbf{I}$ is the identity matrix and $\mathbf{T}=\nabla \mathbf{u}+(\nabla \mathbf{u})^{\mathrm{T}}$ the stress tensor. By applying the divergence theorem in order to decrease the order of differentiation and to project the natural boundary conditions for heat flux and stress at the boundaries of the domain, equations (24) and (25) reduce to

$$
\begin{aligned}
R_{\mathrm{M}}^{i} & =\int_{V}\left[\left(\mathbf{u} \cdot \nabla \mathbf{u}+\frac{1}{F r} \mathbf{k} T\right) \phi^{i}+\left(-p \mathbf{I}+\frac{1}{R e} \mathbf{T}\right) \cdot \nabla \phi^{i}\right] \mathrm{d} V-\int_{\mathrm{S}} \mathbf{n} \cdot\left(-p \mathbf{I}+\frac{1}{\operatorname{Re}} \mathbf{T}\right) \phi^{i} \mathrm{~d} S=\mathbf{0}, \\
R_{\mathrm{E}}^{i} & =\int_{V}\left(\mathbf{u} \cdot \nabla T \phi^{i}+\frac{1}{P e} \nabla T \cdot \nabla \phi^{i}\right) \mathrm{d} V-\frac{1}{P e} \int_{S} \mathbf{n} \cdot \nabla T \phi^{i} \mathrm{~d} S=0 .
\end{aligned}
$$

Since essential boundary conditions for $u, v$ and $T$ will be applied to all but the outflow boundary of the domain, equations (26) and (27) will be replaced by equations (8)-119) accordingly. Additionally, for the SBFS problem, since $\partial T / \partial x=0$ at the lower half of the entrance, the surface integral of the energy equation at this boundary is identically zero. Consequently, the surface integrals in equations (26) and (27) still need to be evaluated or replaced or handled in some way at the synthetic outflow according to equations $(20)-(22)$ or the free boundary condition.

The residuals are evaluated numerically by a nine-point Gaussian integration. A system of non-linear algebraic equations results, which is solved by Newton iteration according to the scheme

$$
\mathbf{q}^{(n+1)}=\mathbf{q}^{(n)}-\mathbf{J}^{-1} \mathbf{R}\left(\mathbf{q}^{(n)}\right)
$$

where $\mathbf{q}=\left[u_{1}, v_{1}, T_{1}, p_{1}, \ldots, u_{N}, v_{N}, T_{N}, p_{N}\right]$ is the vector of the unknowns and $\mathbf{J}=\partial \mathbf{R} / \partial \mathbf{q}$ is the Jacobian matrix of the residuals $\mathbf{R}$ with respect to the nodal unknowns $\mathbf{q}$. The banded matrix of the resulting linear equations is solved by a frontal solver ${ }^{13}$ at each Newton iteration.

\section{RESULTS}

The stratified backward-facing step problem (SBFS problem, Figure 2(b))

First the fully developed flow boundary condition, equations (20) and (21), at the synthetic exit was imposed on a long domain truncated to 15 units of length. With $F r=16 / 9$ and $\operatorname{Pr}=1$ a solution was obtained initially at $R e=1$ and zero-order continuation was used to reach $R e=800$. The Newton iteration converged quadratically within four iterations and the $R e-$ number was increased by 50 each time a solution was obtained. The predicted $u$-velocity profiles of the final solution are plotted in Figure 4(a). It is obvious that the flow was not fully developed up to $x=14 \cdot 5$; therefore the assumption of a fully developed parabolic $u$-velocity profile at the exit imposes an artificial constraint on the system that violates the continuity of the flow in the domain. The results obtained do not predict well the behaviour of the flow near the exit of the flow, as shown in Figure 4(a). Since one is uncertain about the actual physics of the problem at the synthetic outflow, guessing a probable boundary condition (here the fully developed flow) may distort physical reality. Other traditional boundary conditions, of zero axial stress or total force, result in the same ambiguities.

A second approach to the same problem was taken by simply evaluating the surface integral of equations (26) and (27) at the outflow along with the volume integrals of the same equations. This is equivalent to extending the validity of the weighted momentum equation to the synthetic outlet 

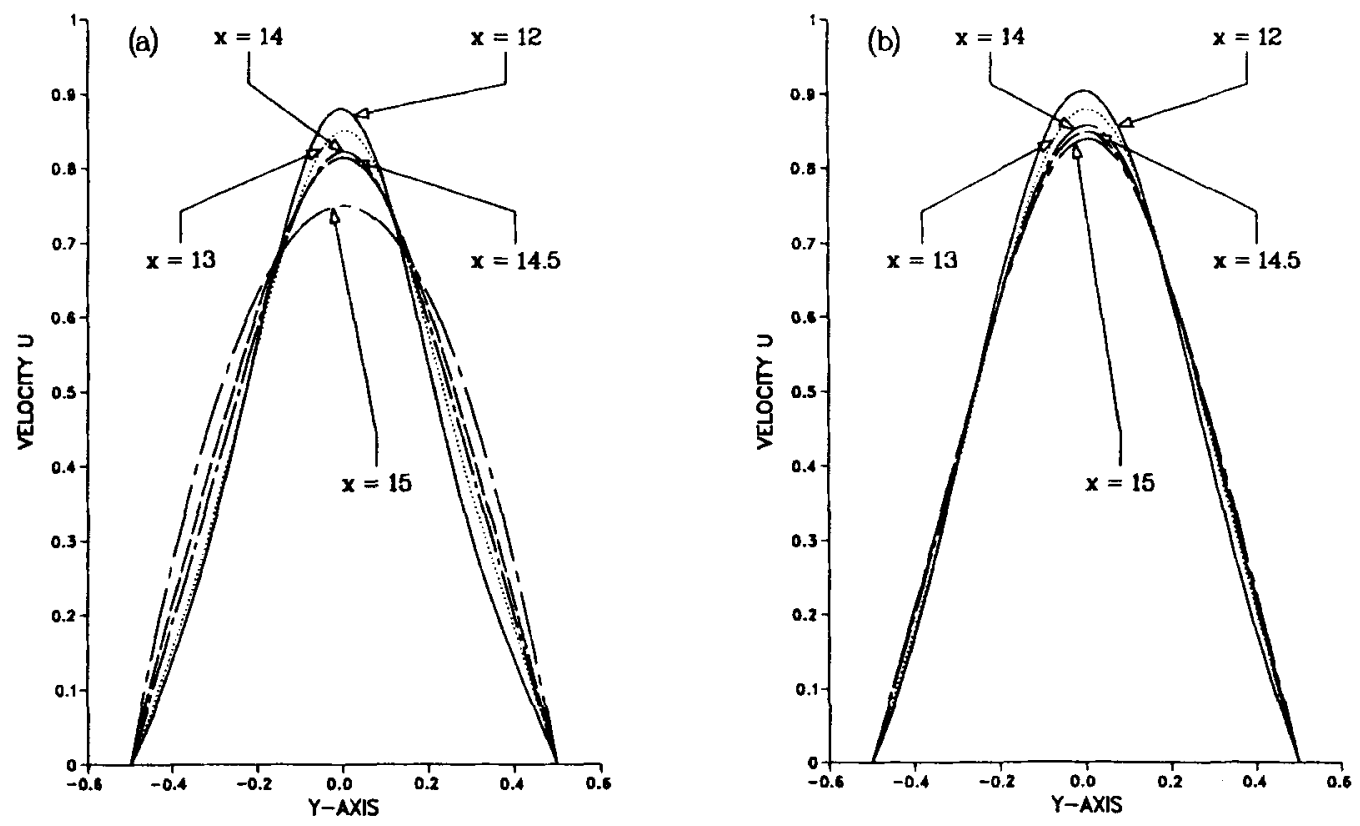

Figure 4. Predicted axial velocity profiles near exit of long domain for (a) fully developed flow and (b) free boundary condition applied at synthetic outflow at $x=15$

instead of replacing it there by risky, unknown essential (or natural) boundary conditions. This is the free boundary conditions. The initial guess was the solution obtained with fully developed flow boundary conditions at the exit. The Newton iteration converged in five iterations to the solution (Figure 4(b)). The same solution was also obtained with zero-order continuation starting from $R e=1$. The results indicate that the assumption of fully developed flow at the exit predicts rather well the flow behaviour up to eight units upstream from the exit of the long domain. Comparisons of the results obtained with the two different boundary conditions are shown in Figures 5 and 6 . As demonstrated in Figure 4, the error in the $u$-velocity profile near the exit is alleviated by the free boundary condition, which extends the validity of the weighted momentum equation to the synthetic outflow without imposing a fully developed flow there. The predicted pressures in Figure 5 also show that the fully developed flow assumption was unfortunate as opposed to the smooth pressure distribution when the free boundary condition was applied.

For the energy equation two outflow boundary conditions have been tested: (a) equation (22) and (b) the free boundary condition, equation (27). In both cases identical results have been obtained, as depicted in Figure 7. Consequently, the assumption of negligible heat conduction in the $x$-direction far downstream from the entrance of the domain $(x \geqslant 15)$ is physically correct.

As shown by Figure 6, a short truncated domain $(x=7)$ will cut the development of an eddy. Unlike the case of the long domain, a solution that assumes fully developed flow at the synthetic exit would be quite inappropriate. Consequently, only a solution with the free boundary condition was sought in the case. Again, zero-order continuation was used starting from $R e=1$ to advance to the final solution at $R e=800$. The results of this trial are compared with the corresponding results obtained with the long domain in Figures 8-10. Apart from an error of up to $20 \%$ in the pressure alone, right at the outlet, the predictions are satisfactory. It is obvious that the free boundary condition at the synthetic outflow preserves the actual physics of the flow 

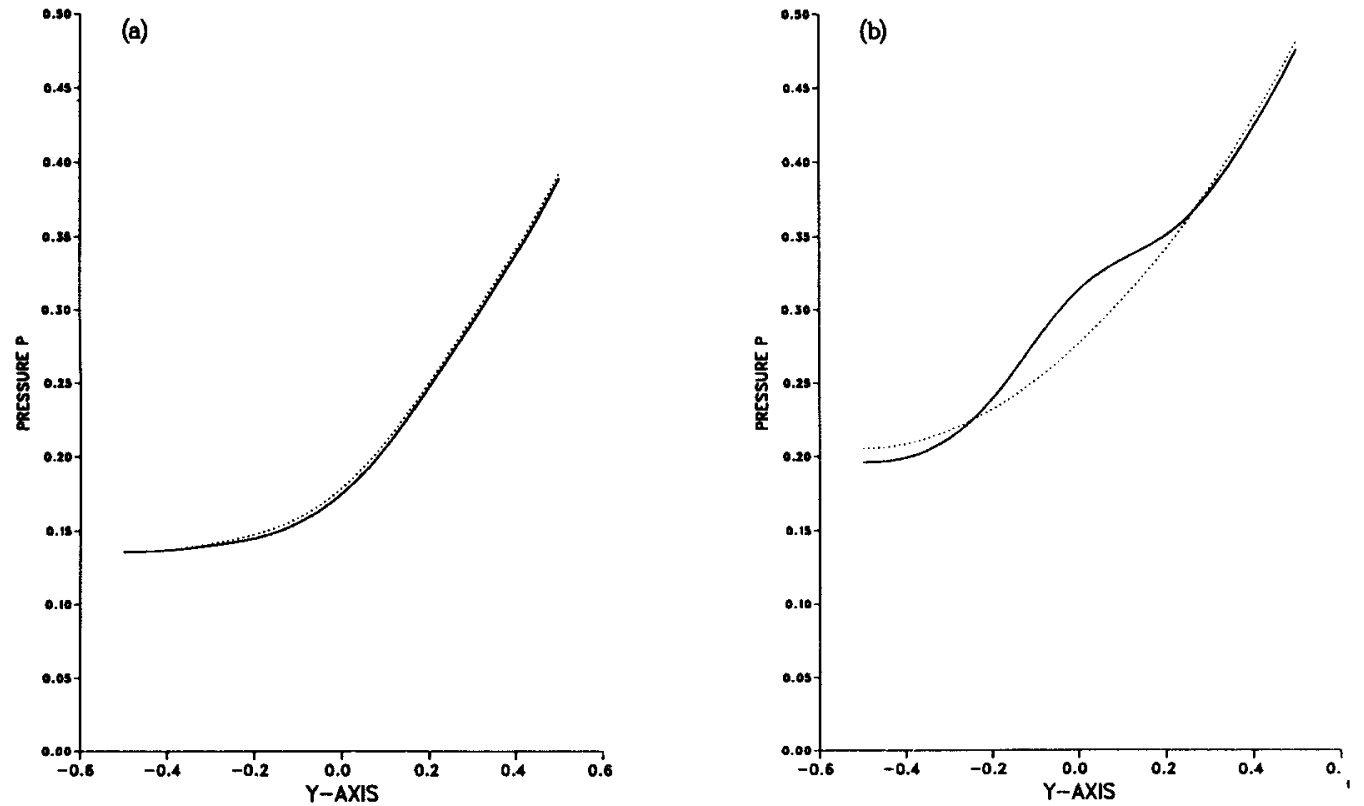

Figure 5. Comparison of pressure distributions across channel at (a) $x=7$ and (b) $x=15$ predicted with fully developed flow $(-)$ and free boundary condition $(\cdots)$ with long domain $0 \leqslant x \leqslant 15$
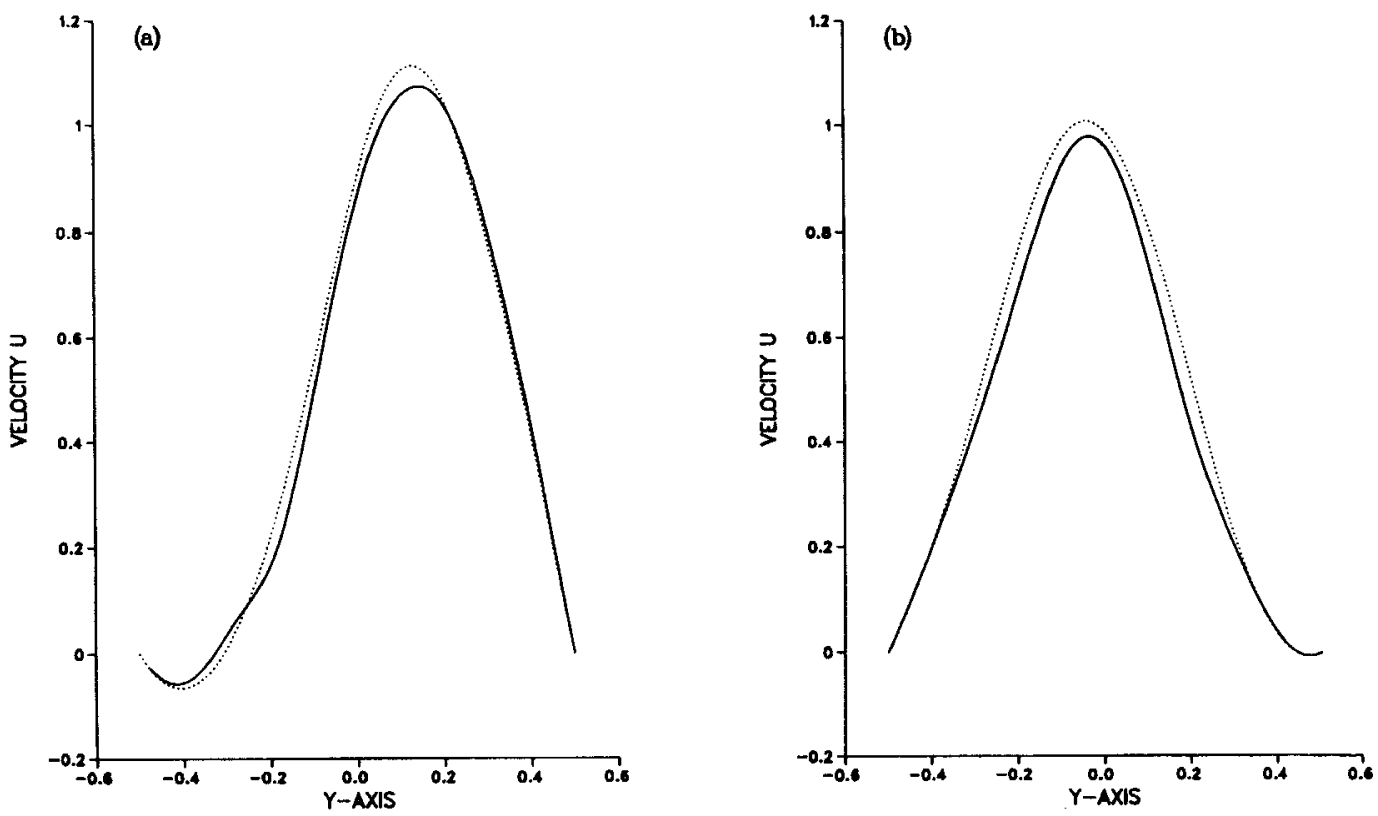

Figure 6. Comparison of $u$-velocity distributions across channel at (a) $x=7$ and (b) $x=9$ predicted with fully developed flow $(\longrightarrow)$ and free boundary condition $(\cdots)$ with long domain $0 \leqslant x \leqslant 15$ 

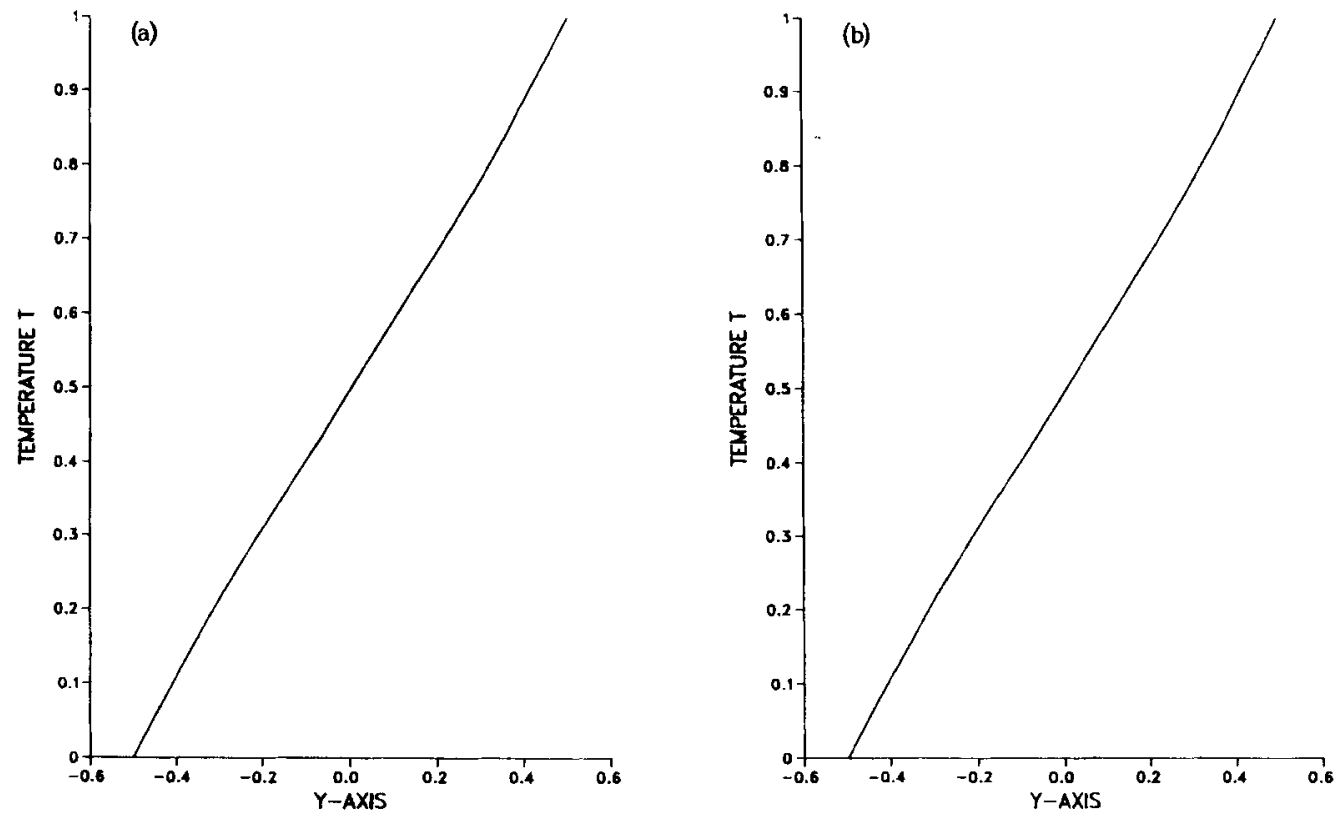

Figure 7. Temperature profiles across channel at (a) $x=14$ and (b) $x=15$ predicted by both essential and free boundary conditions with long domain $0 \leqslant x \leqslant 15$
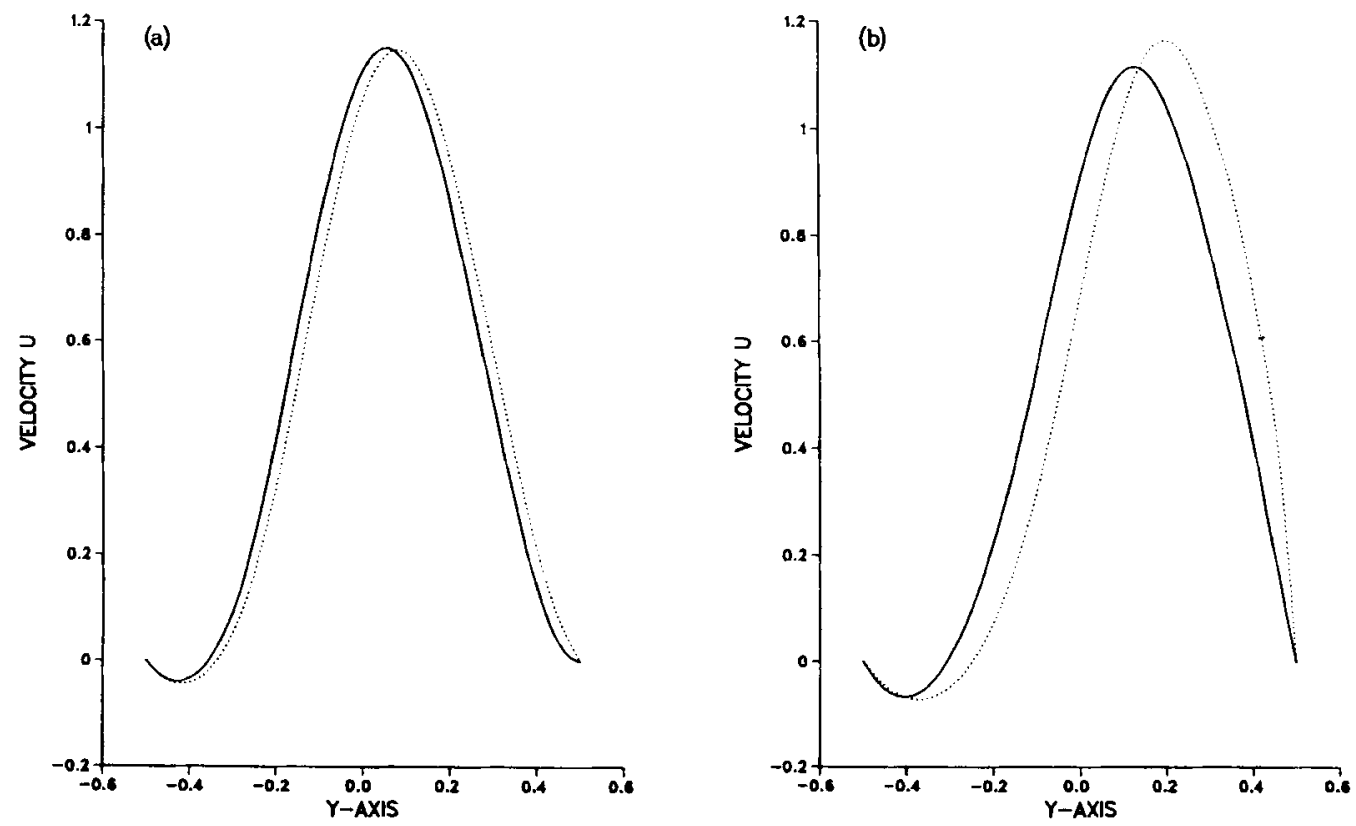

Figure 8. Comparison of $u$-velocity distributions across channel at (a) $x=5.5$ and (b) $x=7$ predicted with free boundary condition applied at $x=15(\longrightarrow)$ and $x=7(\cdots)$ 

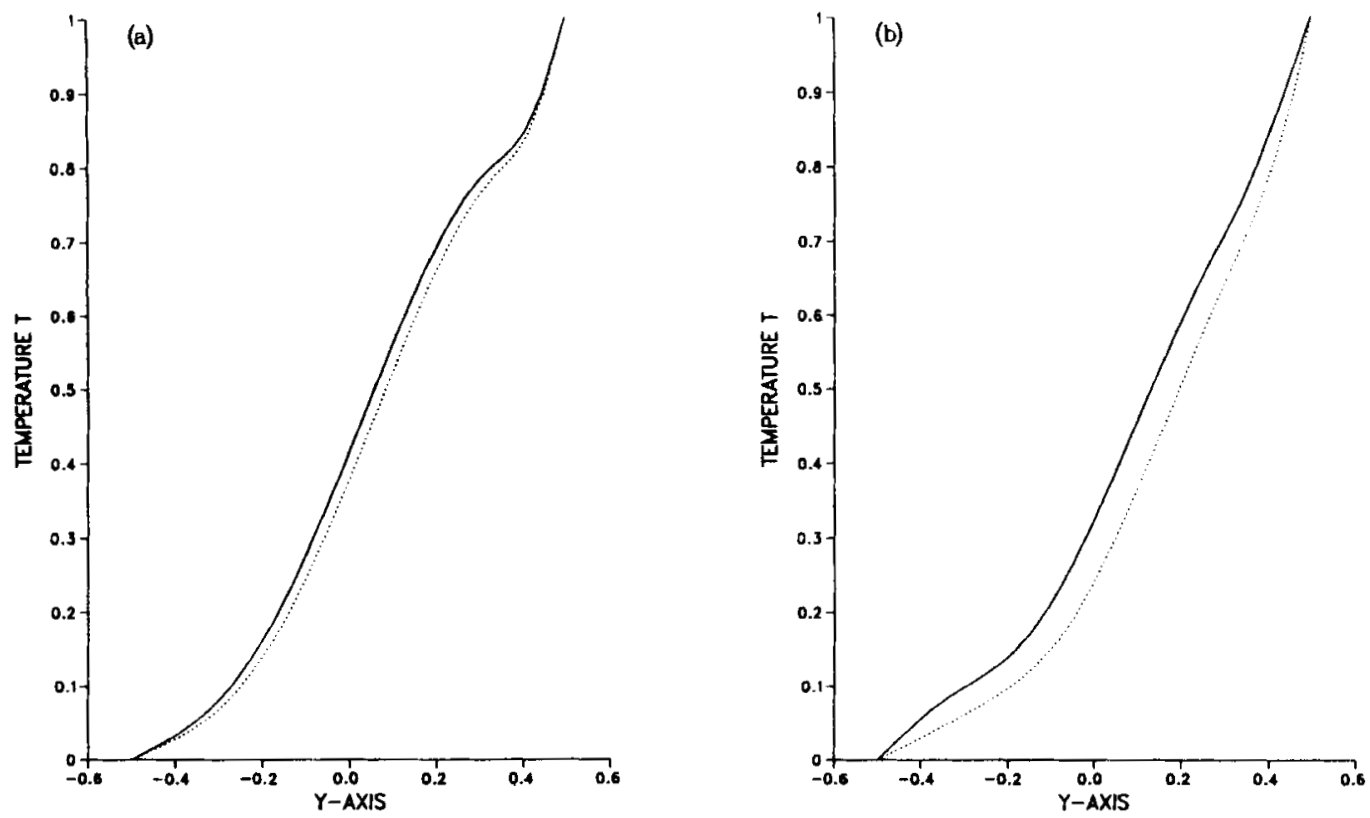

Figure 9. Comparison of temperaure distributions across channel at (a) $x=5 \cdot 5$ and (b) $x=7$ predicted with free boundary condition applied at $x=15(-)$ and $x=7(\cdots)$
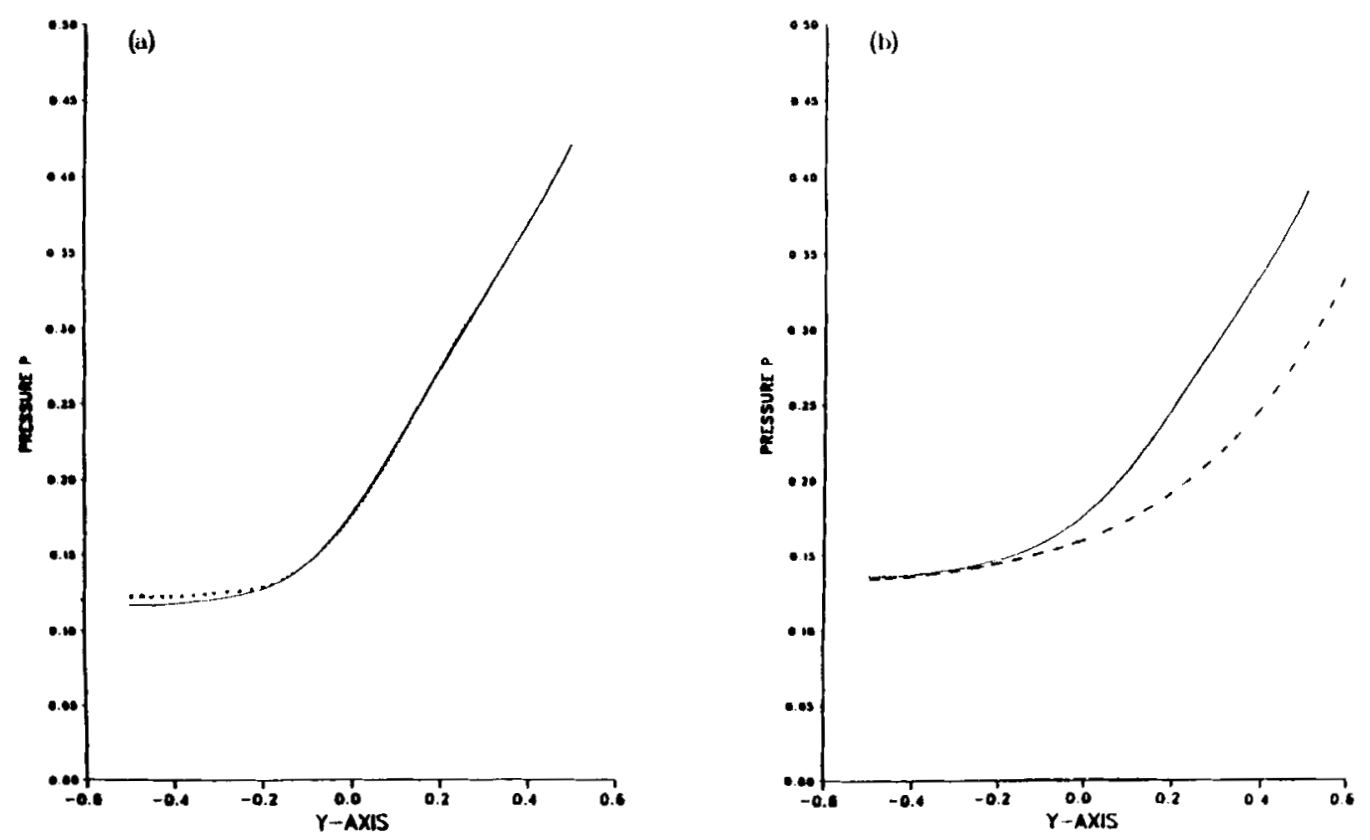

Figure 10. Comparison of pressure distributions across channel at (a) $x=5 \cdot 5$ and (b) $x=7$ predicted with free boundary condition applied at $x=15(-)$ and $x=7(\cdots)$ 
within a certain margin of error. This error is not decreased when the mesh is further refined. As shown in Figure 11, the velocity and temperature profiles do not change significantly when the fine mesh of Figure 3(b) is used, since the solution obtained with the truncated 'typical' computational domain (Figure 3(a)) is mesh-independent. In Figure 12 the streamline patterns obtained from this solution are compared with the benchmark solution obtained with $80 \times 480=38400$ elements, as communicated by Leone. ${ }^{14}$ The agreement is remarkable.

The backward-facing step problem (BFS problem, Figure 2(a))

In order to determine the validity of the outflow boundary conditions, a standard solution for $R e=800$ was calculated. The validity of the tested experimental boundary conditions was determined by 'truncating' the standard mesh and applying the boundary condition at the new synthetic outflow plane. No grid rearrangement was performed when the domain was truncated because a boundary condition that truly reflects the flow conditions at this point should converge to the standard solution; any other results were just accepted 'as is'. All finite element computations were performed using the mesh illustrated in Figure 3(a).

The standard solution was obtained by applying the fully developed outflow boundary conditions on successively longer and longer computational domains. As the domain length increases, the flow in close proximity to the exit should reach fully developed (Poiseulle) flow with a constant pressure gradient. The continuation method started from $R e=1$ and continued up to $R e=800$. The results for the standard solution $(x=35)$ are illustrated in Figure 13. The pressure distributions along the top, centre and bottom collapse into a single line at a downstream location of 15 width units, indicating a constant cross-channel pressure distribution. The flow
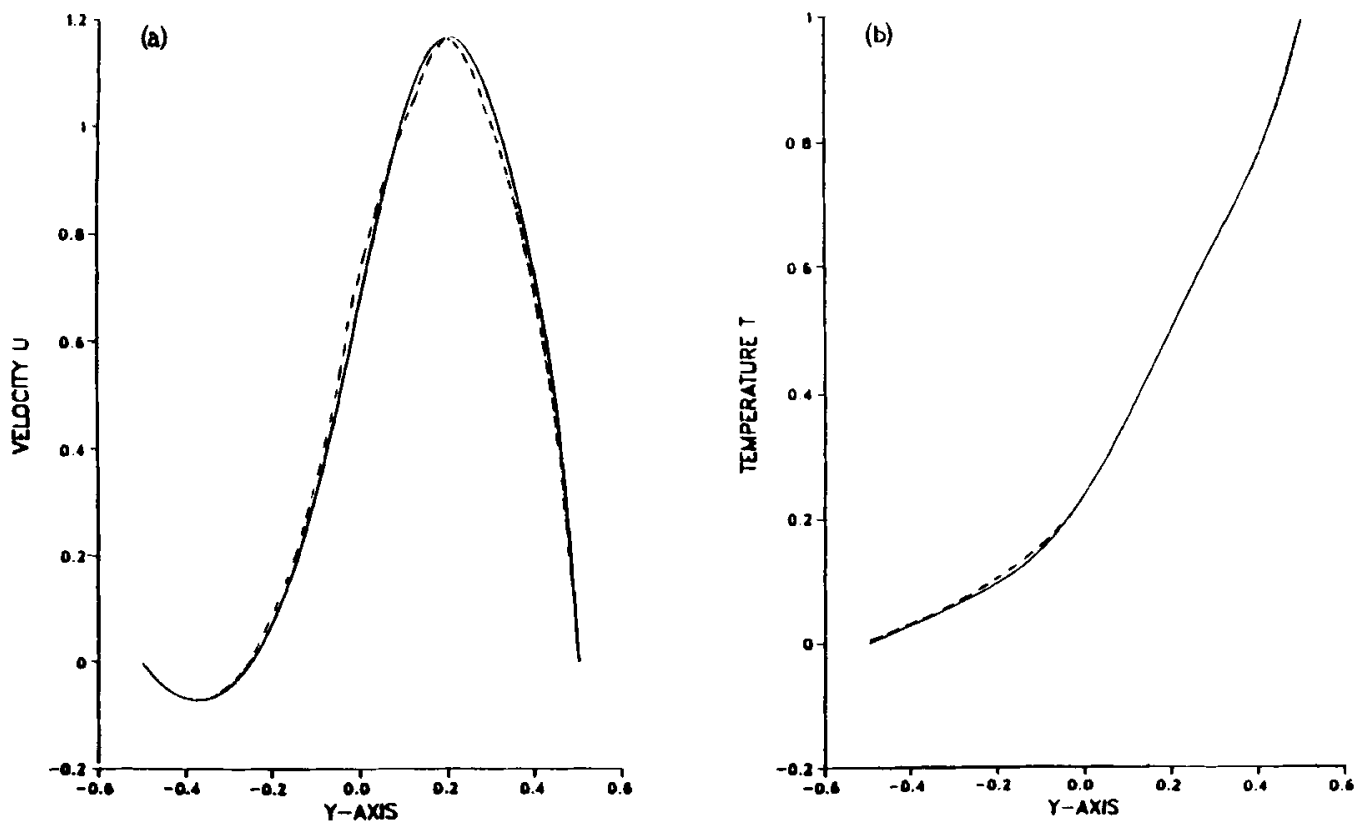

Figure 11. Comparison of (a) velocity and (b) temperature profiles at $x=7$ using truncated 'typical' domain of Figure 3(a) $(-)$ and refined $46 \times 18$ domain of Figure $3(b)(--)$ 
(a)

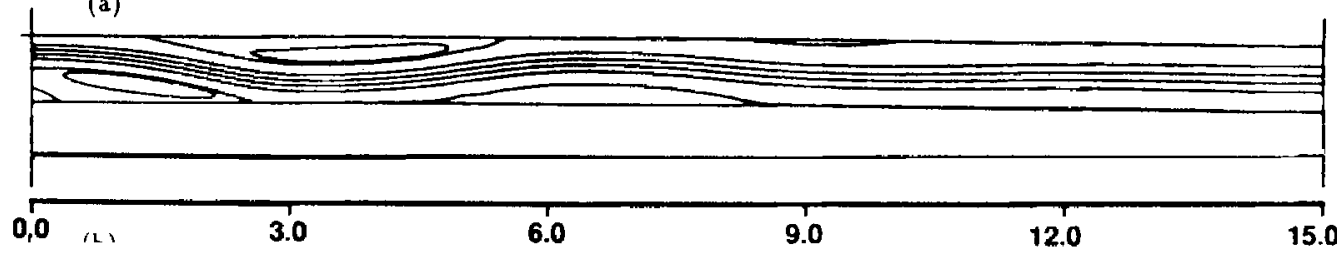

(b)

DISTANCE FROM INLET

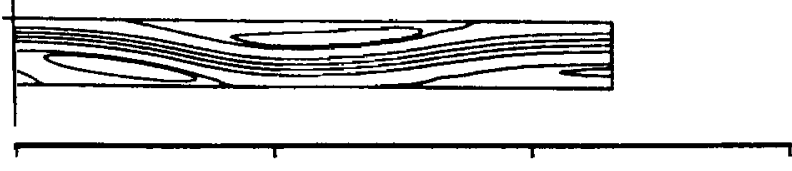
0.0
3.0
6.0
9.0

(c)

DISTANCE FROM INLET

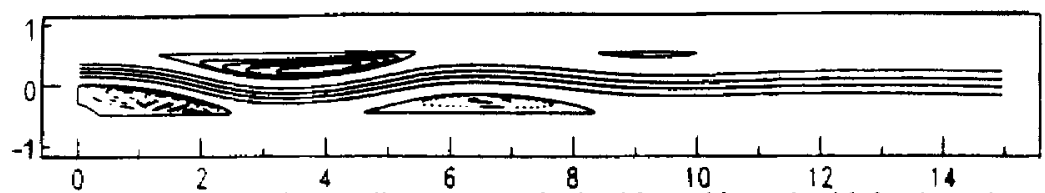

Figure 12. Comparison of streamline patterns obtained from this work with free boundary condition applied at (a) $x=15$ and (b) $x=7$ for SBFS problem with data given in benchmark solution ${ }^{14}$

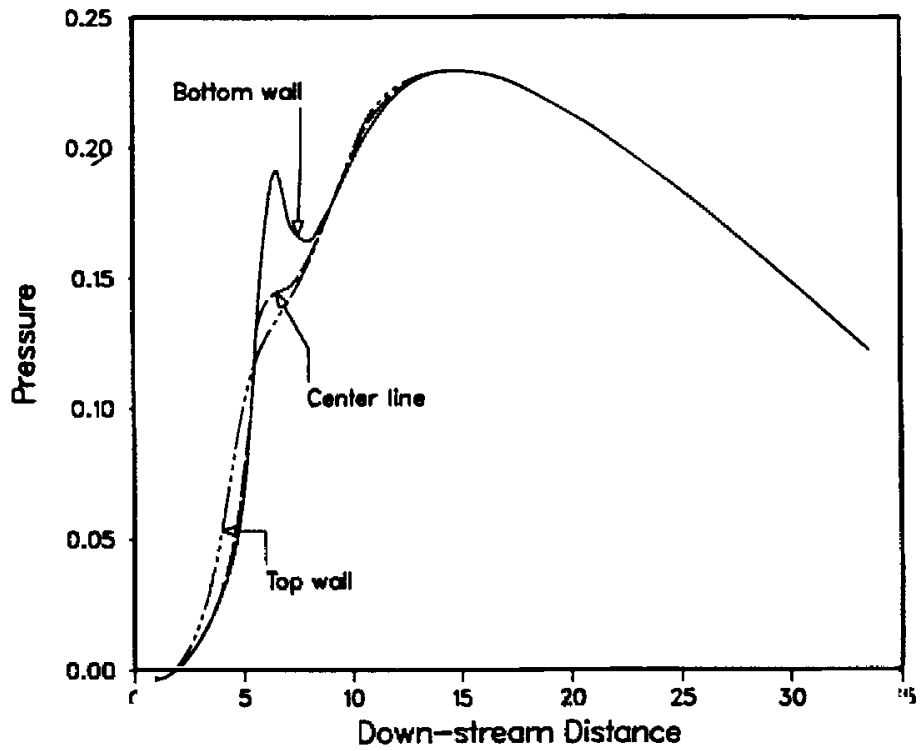

Figure 13. Predicted pressure distributions for standard solution on domains extending to $x \geqslant 30$ where flow is fully developed 
becomes truly fully developed though at a downstream location of approximately $x=27$, which is signified by the onset of a linear pressure drop.

$\mathrm{BC} 1$ is simply the assumption of fully developed flow at the synthetic exit. This boundary condition was applied at $x=15$ where the flow assumes a parallel configuration. Figure 14 compares the outflow velocity profiles with that of the standard solution. The standard solution shows a much higher $u$-velocity in the centre and lower values near the walls when compared with the result with $\mathrm{BC} 1$. This occurs because the flow has not recovered from the effects of the low fluid velocities in the two eddies located at the top and bottom walls. This is also seen in the pressure profiles which are plotted in Figure 15. The standard solution has a constant pressure profile at the outlet, while the $\mathrm{BCl}$ solution produces a profile with an increased pressure at the centre which serves to decelerate the flow to the assumed fully developed velocity profile. The $u$-velocity component at $x=7$ and 3 for the standard solution and that produced by $\mathrm{BC} 1$ applied at $x=15$ also show differences, as do the pressure profiles illustrated in Figures 14 and 15 . As pointed out earlier, the flow at $x=15$ is not fully developed and this assumption clearly violates the physics of the flow. This fact is illustrated in Figure 16, where the centreline $u$-velocity is compared with that of the standard solution. The artificial enforcement of $\mathrm{BC} 1$ at $x=15$ produces the 'wiggle' in the $u$-velocity.

The condition specified in $\mathrm{BC} 2$, the free boundary condition, is to calculate the surface integral that corresponds to the outflow plane in terms of the, as of yet, unknown boundary nodal values for $u, v$ and $p$. This condition was applied at the synthetic planes corresponding to $x=15$ and 7 . Figure 17 illustrates the excellent agreement of the $u$-component of velocity with that of the standard solution. The pressures shown in Figure 18 agree equally well with the standard solution pressures. The synthetic plane placed at $x=7$ cuts through an eddy at the top wall and is challenging to obtain an accurate solution. Zero-order continuation was used to calculate the flow field with $\mathrm{BC} 2$ applied at $x=7$ starting with $R e=1$. Quadratic convegence was attained in all

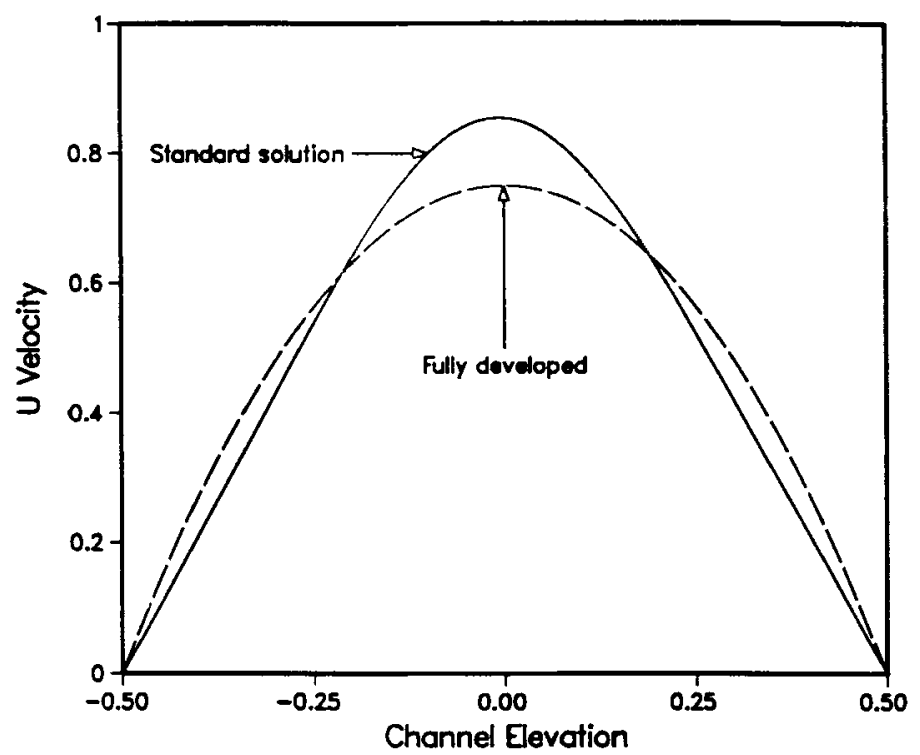

Figure 14. Predicted axial velocity profile at $x=15$ with fully developed flow boundary condition applied at $x=15$ compared with that of standard solution 


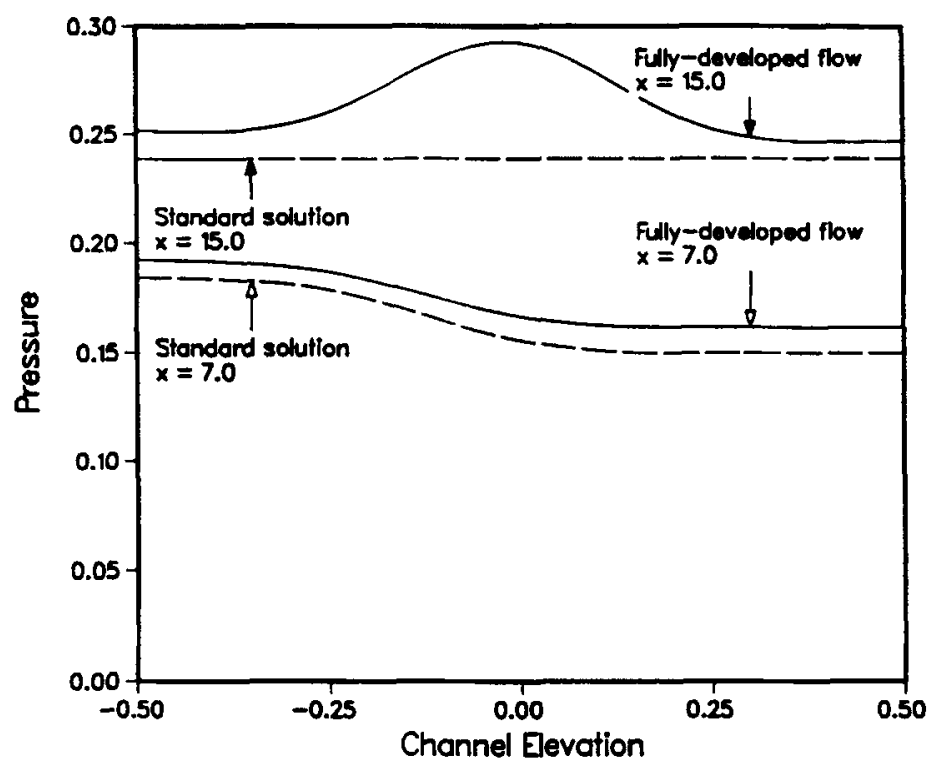

Figure 15. Predicted vertical pressure profiles with fully developed flow boundary condition applied at $x=15$ compared with those of standard solution

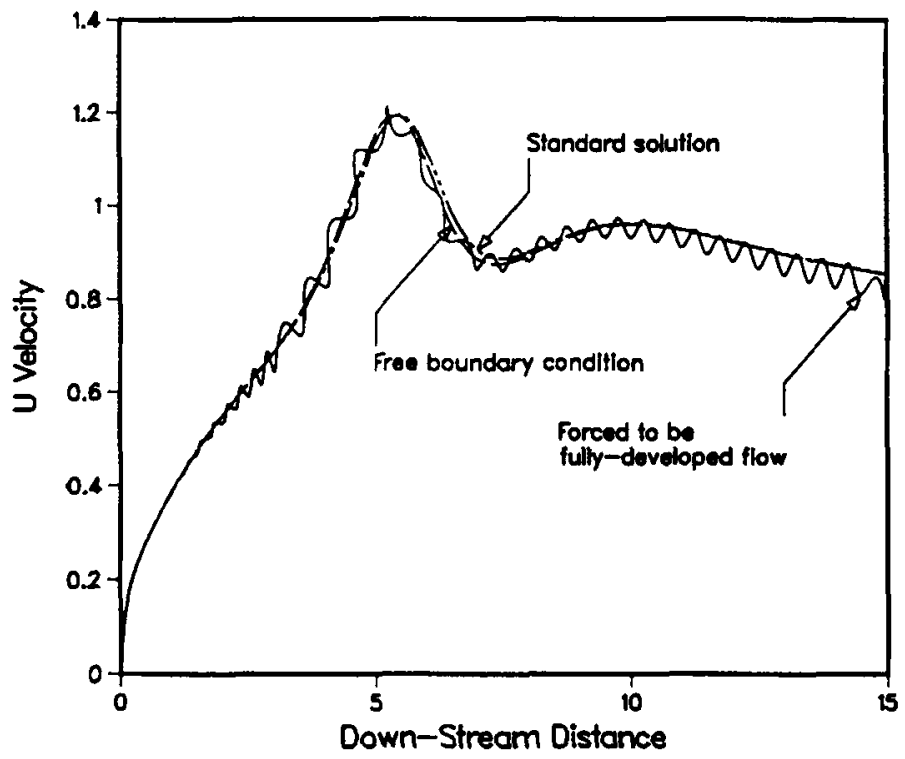

Figure 16. Comparison of centreline $u$-velocity component of standard solution with corresponding ones obtained with fully developed flow boundary condition and with free boundary condition both applied at $x=15$

computations except at extreme values of $R e=750$ where convergence degraded to linear. This was not the case with the SBFS problem, perhaps owing to the fact that at high Reynolds numbers the BFS flow is closer to ideal and therefore more unstable. Despite this problem, accurate results were obtained as shown in Figure 19, where a comparison is made of velocity 


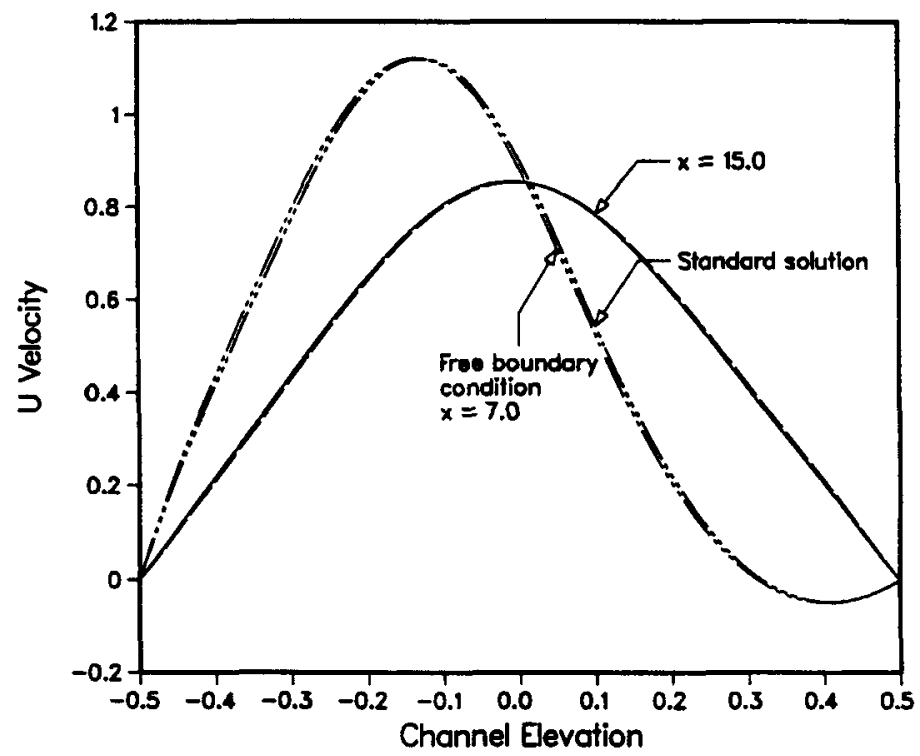

Figure 17. Predicted exit vertical velocity profiles obtained with free boundary condition applied at $x=15$ and 7 compared with those of standard solution

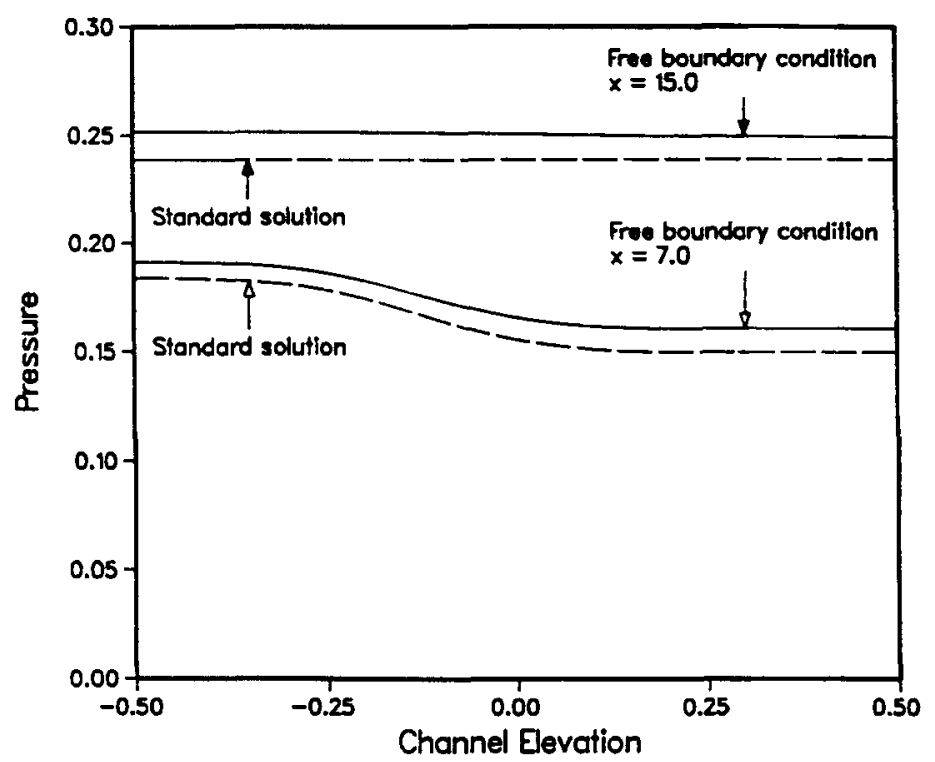

Figure 18. Predicted exit vertical pressure profiles obtained with free boundary condition applied at $x=15$ and 7 compared with those of standard solution

diagrams for both the standard solution and that obtained by BC2. Clearly shown is the formation of the secondary eddy which passes directly through the cutting plane. The attachment and detachment points of the primary eddy at the bottom correlate closely to those of the standard solution. The pressure profile at $x=7$ also agrees with that of the standard solution as indicated by Figure 18. The BFS problem is characterized by the formation of two eddies: 


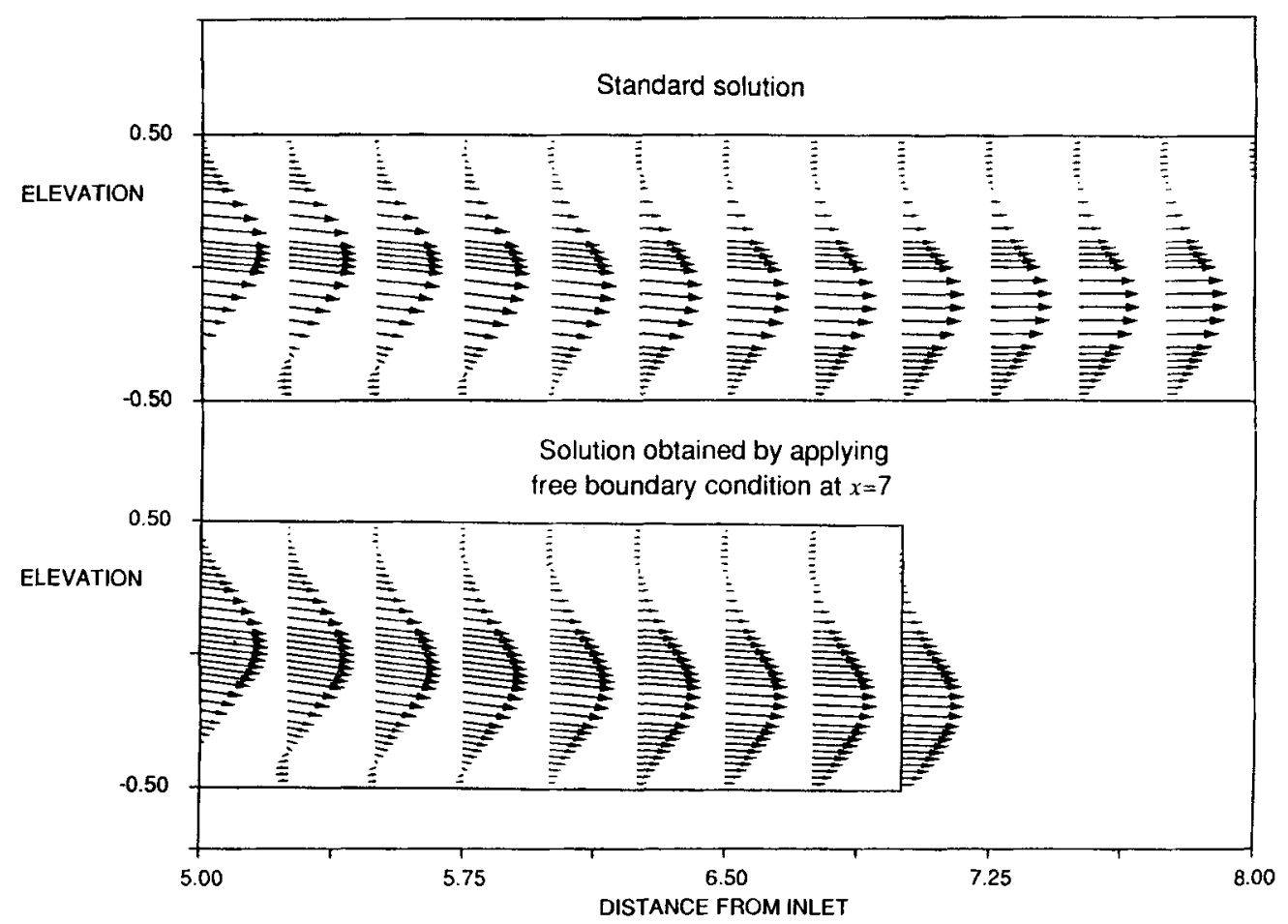

Figure 19. Velocity vector diagrams of standard solution compared with those with free boundary condition applied at $x=7$

a primary eddy is attached to the step and a secondary eddy is attached to the ceiling as illustrated in Figure 20. The primary eddy detaches at the top of the step and attaches at $x=6 \cdot 1$, while the secondary eddy separates at $x=4.8$ and reattaches at $x=10 \cdot 5$. Also shown in Figure 20 are the data of the benchmark solution obtained by Gartling ${ }^{15}$ with 8000 elements. Good agreement exists between the truncated domain of this work and the benchmark solution when streamline $(\psi)$ values are compared.

\section{DISCUSSION}

The answer to why the free boundary condition works is not clear from the pure mathematics point of view. It, may be the non-linearity of the governing equations which can exhibit local ellipticity or hyperbolicity under appropriate conditions. ${ }^{16}$ This seems not to be the case because the same calculations can be made for creeping flow of $R e \rightarrow 0$ as shown in Figure 21. For this example the governing equations are elliptic in the velocity $\mathbf{u}$ and require boundary conditions completely enclosing the domain. One must look more deeply into what is occurring when the Galerkin procedure is applied to the Navier-Stokes equations. In this analysis it is not suggested that no boundary conditions are required in the mathematical sense. Rather, it would appear that the success in applying the free boundary condition is the result of the Galerkin finite element procedure itself. In simplified cases the Galerkin procedure produces the same approximations as, for example, finite difference approximations. However, for problem statements with non-linear terms or multidimensional problems the Galerkin procedure produces a distinct representation. ${ }^{17}$ One may gain some insight to the reason as to why applying the free boundary condition 


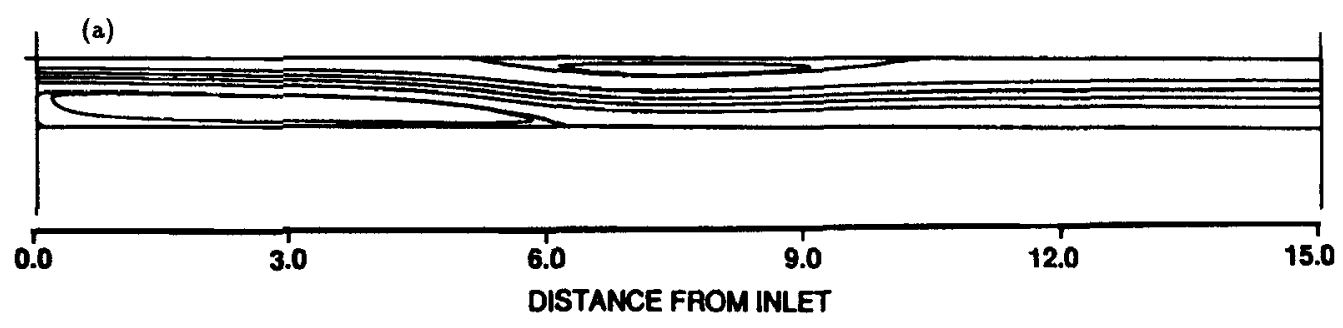

(b)

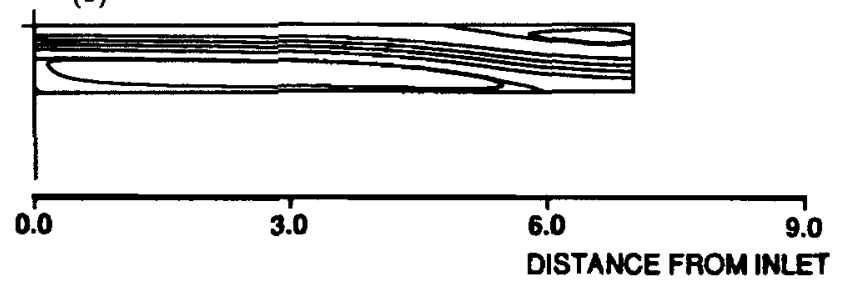

(c)

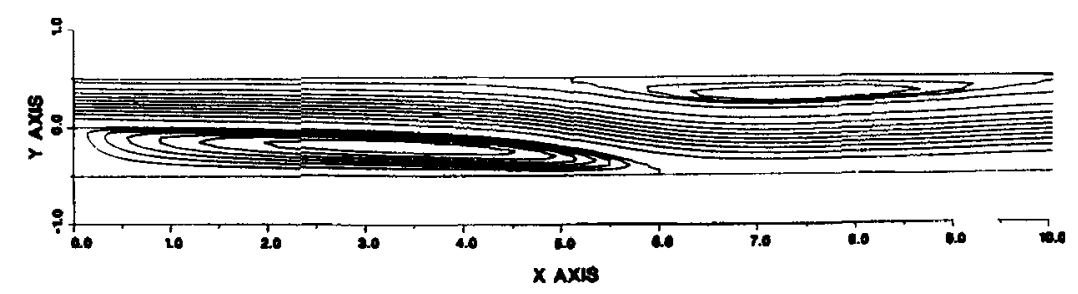

Figure 20. Predicted streamline patterns of standard solution and with free boundary condition applied at (a) $x=15$ and (b) $x=7$ for isothermal BFS problem compared with data given in benchmark solution ${ }^{15}$

works and under what conditions it will work by examining the Stokes creeping flow limit in more detail. The Stokes equations are obtained by minimizing the viscous dissipation function subject to the constraint of mass conservation. This can be stated in a functional form as

$$
I=\int_{\mathbf{\Omega}} \mathbf{T}: \nabla \mathbf{u} \mathrm{d} \boldsymbol{\Omega}+\int_{\mathbf{\Omega}} \lambda \boldsymbol{\nabla} \cdot \mathbf{u} \mathrm{d} \boldsymbol{\Omega},
$$

where $\lambda$ is the Lagrange multiplier which is used to enforce conservation of mass. A quick calculation shows that $\lambda$ is identical to twice the pressure $p$ and the final form of the functional becomes $^{17,18}$

$$
I=\int_{\Omega}(-2 p \nabla \cdot \mathbf{u}+\mathbf{T}: \nabla \mathbf{u}) \mathrm{d} \Omega
$$

To find the corresponding set of partial differential equations which minimize the functional, the Euler-Lagrange equation

$$
\frac{\partial f}{\partial w_{k}}-\nabla \cdot\left(\frac{\partial f}{\partial\left(\nabla w_{k}\right)}\right)=0
$$




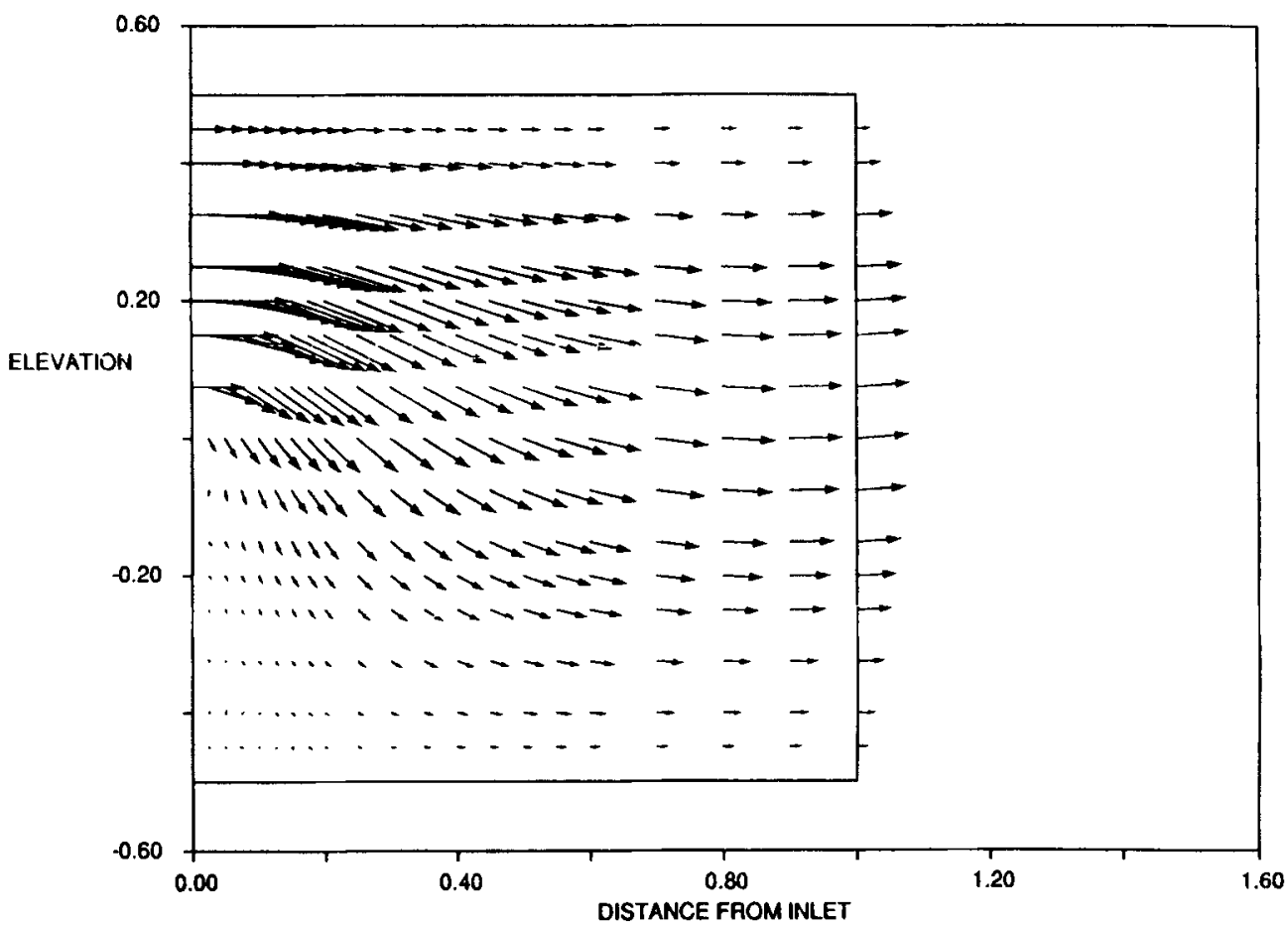

Figure 21. Example of creeping flow calculation for isothermal BFS problem with free boundary condition applied at $x=1$

Table I. Calculated values of the energy functional for various boundary conditions applied at $x=15$

\begin{tabular}{ll}
\hline$u$ and $v$ specified & $I=133.713529$ \\
$\tau_{x x}=0$ and $v=0$ & $I=129 \cdot 376384$ \\
$\tau_{x x}=0$ and $\tau_{x y}$ free & $I=129.332292$ \\
Free boundary condition & $I=129.332111$
\end{tabular}

must be evoked for each of the unknowns $u, v$ and $p$, where $f$ is the integrated of equation (29). This differentiation is straightforward and yields the Stokes limit of the Navier-Stokes equations. Table I shows calculated values for the functional $I$ for various outflow boundary conditions applied at $x=15$. It suggests that among all possible boundary conditions applied at the synthetic boundary, the free boundary condition minimizes the functional $I$, which in cases of real boundaries is minimized by the zero-traction condition. The last two values in Table $I$ are close to one another because it turns out that $\tau_{x x}$ is nearly zero at $x=15$.

Thus it appears that the free boundary condition indeed results in the least value of the functional $I$ (for Stokes flow) and therefore yields the most reasonable solution among all the possible choices at the synthetic outflow. Somehow, the solution obtained with the free boundary condition on a truncated domain mimics the solution on the actual unbounded domain, as evidenced by two different facts: (a) by the fact that the solutions obtained on the truncated 
domain by means of the free boundary condition were almost identical to those obtained by means of the very long domain where the influence of any ouflow boundary condition does not propagate upstream to the truncated domain and (b) by the minimization of the functional. This may suggest that instead of minimizing a functional in a truncated domain subject to essential or natural boundary conditions on every portion of its boundary, including synthetic outlets where the appropriate boundary conditions are not known', it is preferable 'to minimize the functional in the truncated domain and on the synthetic outlet subject to boundary conditions on every portion of the boundary but the synthetic outflow'.

In the finite element formulation these principles apply as follows. For simplicity the differential equation

$$
\boldsymbol{\nabla} \cdot \mathbf{T}=\mathbf{0}
$$

is solved in each finite element of volume $V_{i}$. Then according to the Galerkin method (Section 4) and by applying the divergence theorem, equation (31) becomes

$$
\int_{V_{i}}(\nabla \cdot \mathbf{T}) \phi_{i} \mathrm{~d} V_{i}=-\int_{V_{i}}\left(\mathbf{T} \cdot \nabla \phi_{i}\right) \mathrm{d} V_{i}+\int_{S_{i}}(\mathbf{n} \cdot \mathbf{T}) \phi_{i} \mathrm{~d} S_{i}=0 .
$$

For internal elements the last (boundary) term cancels out during the assembly element by element, because $\phi_{i}$ is common to adjacent elements evaluated at their common boundary and their corresponding $\mathbf{n}$ are opposite to each other. For an element adjacent to the synthetic boundary, for the same reasons, the only surviving boundary term is the one on the synthetic boundary, say $S_{\mathrm{b}}$, and therefore

$$
\int_{V_{i, b}}\left(\mathbf{T} \cdot \nabla \phi_{i}\right) \mathrm{d} V_{i}-\int_{S_{\mathrm{b}}}(\mathbf{n} \cdot \mathbf{T}) \phi_{i} \mathrm{~d} S_{i}=0,
$$

which is essentially the free boundary condition. Equation (33) can also be written, given $\boldsymbol{\nabla} \cdot \mathbf{T}=0$, as

$$
\int_{V_{i, \mathrm{~b}}}\left(\boldsymbol{\nabla} \cdot \mathbf{T} \phi_{i}\right) \mathrm{d} V_{i}-\int_{S_{\mathrm{v}}}\left(\mathbf{n} \cdot \mathbf{T} \phi_{i}\right) \mathrm{d} S_{i}=\mathbf{0} .
$$

The physical significance of the last equation is a relation between the variation of the weighted quantity $\mathbf{T} \phi_{i}$ and its 'flux' through the synthetic outflow of the corresponding boundary element. Thus the entire element $V_{i, b}$ serves as a synthetic boundary zone and it collapses to the actual boundary $S_{\mathrm{b}}$ when the tesselation becomes infinitely fine there.

In light of lacking rigorous mathematical analysis for systems of non-linear equations in general, it cannot be concluded that the free boundary condition will work with any semi-infinite flow, cases of instance being some simple one-dimensional second-order differential equations solved with linear basis functions, e.g. $\ddot{T}+C=0,{ }^{19}$ but not $\ddot{T}-T+C=0$. Also, the free boundary condition addresses flows on semi-infinite domains, where the fluid content may decay hyperbolically, and cannot replace well-defined essential or natural conditions at natural boundaries. The application of the free boundary condition appears to be independent of the order of the basis functions. It can in principle be implemented with finite difference methods too, by evaluating one-sided derivatives at the synthetic boundary in terms of the upstream grid values. Finally, the free boundary condition was found to perform as well with time-dependent semi-infinite flows, where experimentally observed travelling and solitary waves as well as rates of spreading and levelling of liquids on substrates have been predicted. ${ }^{20}$ 


\section{CONCLUSIONS}

At synthetic boundaries it is uncertain which boundary condition may work when the physics of the problem is not known. The extension of the weak form of the momentum equation to such synthetic boundaries, which eliminates the necessity of imposing arbitrary outflow boundary conditions, has already been proven in several problems to give satisfactory results that predict quite well the physical phenomena occurring in these cases. In this work it has been shown that this idea can be successfully applied to two prototype flows which are receiving a great deal of attention in studying open boundary conditions at synthetic outflows. This approach is especially useful when analytical and asymptotic techniques cannot predict the behaviour of the flow far downstream from the synthetic outflow and therefore appropriate boundary conditions at these synthetic boundaries are impossible. The approach was found to work even when the flow is not developed at the synthetic ouflow (i.e. synthetic outflow which cuts a developing eddy), where conventional approaches may fail completely.

The free boundary condition is equivalent to a relation between the variation of a weighted quantity and its 'flux' through the synthetic boundary, which is inherent to the weak finite element solution of the momentum equations. It has been shown that among several alternative open boundary conditions, the free boundary condition, which simply extends the validity of the momentum equations to the synthetic boundaries, minimizes the appropriate energy functional for the unbounded Stokes flow.

\section{ACKNOWLEDGEMENTS}

This research was supported by a grant for exploratory research by the National Science Foundation, Grant CTS-9004373. The authors acknowledge helpful discussions with Dr. P. Gresho, Associate Editor of this journal.

\section{APPENDIX: NOTATION}

$\begin{array}{ll}F r & \text { Froude number } \\ \mathbf{I} & \text { identity matrix } \\ \mathbf{J} & \text { Jacobian matrix } \\ \mathbf{k} & \text { unit vector in direction of gravity } \\ \mathbf{n} & \text { unit normal vector } \\ p & \text { pressure } \\ P e & \text { Peclet number } \\ \mathbf{q} & \text { vector of unknowns in Newton iteration } \\ r & \text { radial direction in cylindrical co-ordinates } \\ R & \text { residual of Galerkin FEM } \\ R e & \text { Reynolds number } \\ T & \text { temperaure } \\ \mathbf{T} & \text { stress tensor } \\ u & \text { fluid velocity in } x \text {-direction } \\ \mathbf{u} & \text { velocity vector of fluid } \\ v & \text { fluid velocity in } y \text {-direction } \\ x, y & \text { Cartesian co-odinate directions } \\ z & \text { axial direction in cylindrical co-ordinates }\end{array}$




\section{Greek letters}

$\begin{array}{ll}\lambda & \text { Maxwell fluid relaxation time } \\ \tau & \text { normal shear stress } \\ \phi & \text { biquadratic basis functions } \\ \psi & \text { bilinear basis functions }\end{array}$

\section{Superscript}

$i \quad i$ th node in FEM

\section{Subscripts}

C continuity equation

E energy equation

M momentum equation

Mathematical symbols

$\partial \quad$ partial derivative

$\nabla \quad$ nabla operator

\section{REFERENCES}

1. H. F. Weinberger, Partial Differential Equations, Blaisdell, London, 1965.

2. A. L. Camerlengo and J. J. O'Brien, 'Open boundary conditions in rotating fluis', J. Comput. Phys., 35, 12 (1980).

3. B. Gustafsson and H. O. Kreiss, 'Boundary conditions for a time dependent problem with artificial boundary', J. Comput. Phys., 30, 333 (1978).

4. K. I. Orlanski, 'A simple boundary condition for unbounded hyperbolic flows', J. Comput. Phys., 21, 251 (1976).

5. P. M. Gresho, R. I. Lee and R. L. Sani, 'On the time-dependent solutions of the incompressible Navier-Stokes equations in two and three dimensions', in C. Taylor and K. Morgan (eds), Recent Advances in Numerical Methods in Fluids, Vol. 1, Pineridge, Swansea, 1979.

6. B. G. Higgins, 'Downstream development of two-dimensional viscocapillary film flow', Ind. Eng. Chem. Fund., 21, 168 (1982).

7. N. E. Bixler, 'Stability of a coating flow', Ph.D. Thesis, University of Minnesota, 1982.

8. S. F. Kistler and L. E. Scriven, 'Coating flow theory by finite elements and asymptotic analysis', Int. j. numer. methods fluids, 4, 207 (1984).

9. T. C. Papanastasiou, 'Coating flows and processing of viscoelastic liquids: fluid mechanics, rheology and computeraided analysis', Ph.D. Thesis, University of Minnesota, 1984.

10. T. C. Papanastasiou, C. W. Macosco and L. E. Scriven, 'Analysis of lubricated squeezing flow', Int. numer. metods fluids, 10, 819 (1986).

11. T. C. Papanastasiou, A. N. Alexandrou and W. P. Graebel, 'Rotating thin films in bell sprayers and spin coating', J. Rheol., 32, 485 (1988).

12. R. D. Wesson and T. C. Papanastasiou, 'Flow singularity and slip velocity in plane extrudate-swell computations', J. Non-Newtonion Fluid Mech., 26, 277 (1988).

13. P. Hood, 'Frontal solution program for unsymmetric matrices', Int. j. numer. methods eng., 10, 379 (1974).

14. J. M. Leone, Jr., 'Open boundary condition symposium. Benchmark solution: stratified flow over a backward facing step', Int. j. numer. methods fluids, 11, 969 (1990).

15. D. K. Gartling, 'A test problem for outflow boundary conditions--flow over a backward-facing step', Int. $j$. numer. methods fluids, 11, 953 (1990).

16. M. Ahrens, Y. J. Yoo and D. D. Joseph, 'Hyperbolicity and change of flow type in the flow of viscoelastic fluids through pipes', J. Non-Newtonian Fluid Mech., 24, 67 (1987).

17. A. J. Baker, Finite Element Computational Fluid Mechanics, McGraw-Hill, New York, 1983.

18. J. J. Connor and C. A. Brebbia, Finite Element Techniques for Fluid Flow, Newnes-Butterworths, London, 1978.

19. P. M. Gresho, Personal communications, May 1991.

20. N. T. Malamataris and T. C. Papanastasiou, 'Unsteady free surface flows on truncated domains', Ind. Eng. Chem. Res., 30, 2211 (1991). 